\title{
Mutual information based CT registration of the lung at exhale and inhale breathing states using thin-plate splines
}

\author{
Martha M. Coselmon, ${ }^{a)}$ James M. Balter, Daniel L. McShan, and Marc L. Kessler \\ Department of Radiation Oncology, University of Michigan, Ann Arbor, Michigan 48109
}

(Received 14 November 2003; revised 15 July 2004; accepted for publication 16 August 2004; published 19 October 2004)

\begin{abstract}
The advent of dynamic radiotherapy modeling and treatment techniques requires an infrastructure to weigh the merits of various interventions (breath holding, gating, tracking). The creation of treatment planning models that account for motion and deformation can allow the relative worth of such techniques to be evaluated. In order to develop a treatment planning model of a moving and deforming organ such as the lung, registration tools that account for deformation are required. We tested the accuracy of a mutual information based image registration tool using thin-plate splines driven by the selection of control points and iterative alignment according to a simplex algorithm. Eleven patients each had sequential CT scans at breath-held normal inhale and exhale states. The exhale right lung was segmented from CT and served as the reference model. For each patient, thirty control points were used to align the inhale CT right lung to the exhale CT right lung. Alignment accuracy (the standard deviation of the difference in the actual and predicted inhale position) was determined from locations of vascular and bronchial bifurcations, and found to be 1.7, 3.1 , and $3.6 \mathrm{~mm}$ about the RL, AP, and IS directions. The alignment accuracy was significantly different from the amount of measured movement during breathing only in the AP and IS directions. The accuracy of alignment including thin-plate splines was more accurate than using affine transformations and the same iteration and scoring methodology. This technique shows promise for the future development of dynamic models of the lung for use in four-dimensional (4-D) treatment planning. (C) 2004 American Association of Physicists in Medicine. [DOI: 10.1118/1.1803671]
\end{abstract}

\section{INTRODUCTION}

As radiotherapy has become more conformal, geometric uncertainties relating to patient setup as well as breathing and organ motion have become an important issue. The inclusion of respiratory motion in treatment planning has been accomplished in a variety of ways, starting with the definition of the planning target volume and internal target volume. ${ }^{1}$ Other methods include dose convolution and fluence convolution. ${ }^{2-6}$ In each, information about respiratory motion is needed for implementation. Image registration techniques that account for deformation and motion between different breathing states are essential to building dynamic organ models for use in treatment planning and dose calculations. Techniques like respiratory gating are also available to reduce the effects of breathing motion during treatment. ${ }^{7-9}$ To investigate the virtues of such techniques, it will be necessary to apply geometric models that account for motion. Prior investigators have examined the ability of deformable registration to aid in multimodality image fusion and this work has led to the use of deformable methods in "4-D" image registration. ${ }^{10-21}$ Meyer et al. demonstrated the accuracy of mutual information based thin-plate spline warping applied to multimodal data sets in the brain and thorax. ${ }^{11}$ Brock et al. recently explored the use of thin-plate spline (TPS) warping to deform models of the liver between inhale and exhale states sampled from breath-held CT scans. ${ }^{19}$ McLeish et al. have examined rigid as well as deformable image registration to quantify cardiac motion due to breathing. ${ }^{20}$ Additional work by Brock et al. has demon- strated the clinical significance of incorporating respiratory induced liver deformation into dose calculations. ${ }^{21}$ Work in tumor tracking in the lung by both Chen et al. and Seppenwoolde et al. has stressed the importance of incorporating respiratory motion into treatment planning for lung cancer patients. $^{22,23}$ It is likely that a deformable image registration method will be necessary to model the respiratory motion of the lung. Thin-plate splines have been demonstrated to be an effective interpolant for anatomic images, and the use of mutual information as a metric for image registration has proven to be robust and accurate for alignment of volumetric image data. ${ }^{10-13,15,18,19,26}$ Our purpose in this study is to evaluate the use of mutual information based registration with thin-plate splines to model deformation of CT representations of the lung between inhale and exhale breathing states.

\section{METHODS AND MATERIALS}

\section{A. Patient data}

Sequential computed tomography (CT) scans were obtained on a helical CT scanner (CT/I, General Electric, Milwaukee, Wisconsin) for 11 patients. The first scan was obtained at normal exhale followed immediately by a scan at normal inhale. Scans were acquired during coached voluntary breath-hold periods of $18-35$ seconds. The images were reviewed by an expert to ensure the lack of breathing-related artifacts. ${ }^{24}$ Scans were obtained with a pitch of 2 , using a $5 \mathrm{~mm}$ aperture. The total time spent from the start of the first scan through the completion of the second scan was less than 5 minutes. 


\section{B. Registration method}

A mutual information (MI) based registration tool has been developed in-house and allows the use of thin-plate splines as an interpolant to model deformation. In this software, control points are placed manually on a reference and homologous image dataset. The number of control points ("knots") define the amount of deformation possible in transforming the model of local anatomy between the datasets. During the alignment process, the positions of the homologous points are iteratively manipulated by the Nelder-Mead simplex algorithm. For each test configuration, the deformed homologous dataset is compared to the reference dataset by a calculation of the MI. The mutual information of two image data sets, $X$ and $Y$, can be defined as

$$
\operatorname{MI}(X, Y)=\sum_{x, y} p(x, y) \log _{2} \frac{p(x, y)}{p(x) p(y)}
$$

where $p(x, y)$ is the joint distribution of the intensity levels of the two datasets and $p(x) p(y)$ represents the joint distribution of independence of the intensity levels of the two datasets.

The point configuration that maximizes the MI is selected to be the optimal transformation between the two patient models. This alignment tool is used in the present study with 30 control points to align the inhale CT model of a right lung to the corresponding exhale CT model of the right lung. In this study, the exhale CT scan will serve as the reference dataset and the inhale CT scan will serve as the homologous dataset.

To restrict the alignment to the right lung only, masking was done of the CT information to include only the right lung plus a $5 \mathrm{~mm}$ margin. Masking of the reference dataset was accomplished using automatically segmented and manually verified lung contours defined for treatment planning. Masking of the homologous dataset was unnecessary, as the MI algorithm is inherently designed to preferentially score configurations presenting similar features.

Thirty control points were chosen to provide sufficient degrees of freedom for the alignment. Meyer et al. and Brock et al. have shown, for MI-based thin-plate spline registrations, that 24 control points are sufficient to map liver shape changes between CT scans acquired at different times for the same patient. ${ }^{18,19}$ In the current study, the 30 control points were placed to have 5 each on 6 specified IS planes in the exhale dataset. Four points on each plane were placed around the border of the lung and one placed in the interior. To serve as an initial estimate for the inhale control points, the points on the most inferior and second most inferior planes were shifted $2 \mathrm{~cm}$ and $1 \mathrm{~cm}$ inferiorly, and all points were shifted $1 \mathrm{~cm}$ anteriorly. These changes were chosen based on the general breathing displacements observed in the small patient cohort and adjustments were done automatically by the computer after choosing the reference control points. The homologous points were not placed to correspond exactly to the exhale control points in an effort to reduce any registration bias. An example of the control point placement on both the segmented reference exhale CT is shown in Fig. 1.

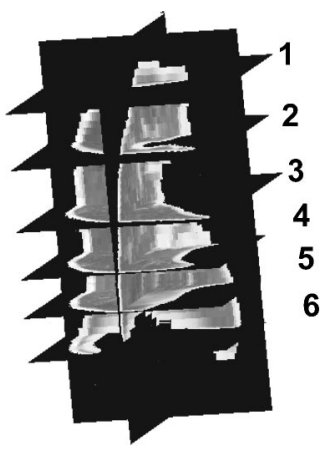

(A)

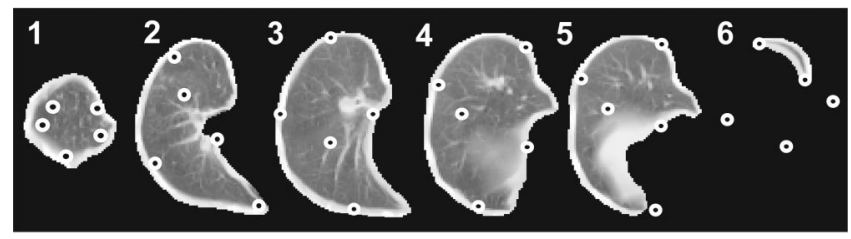

(B)

FIG. 1. Placement of the 30 control points in the cropped reference exhale dataset, showing (a) the six planes where the points are placed, and (b) the 5 points placed in each plane for one representative patient.

To analyze the method dependence on point placement, thin-plate spline registrations were also done with 30 manually selected corresponding exhale-inhale control point pairs as well as a single control point set of 30 pseudo-randomly placed points, used as the control for both datasets. For the manually selected points, the 30 control points were placed consistently in each scan with approximately 20 points near the border of the right lung and 10 points on the interior of the lung. Points were selected while scanning through axial slices in both datasets simultaneously. A point was first chosen on the reference dataset and then the roughly corresponding point was chosen on the homologous dataset. The homologous point did not have to lie on the corresponding slice position. The process of manually selecting control points took approximately 5 minutes assuming the user was experienced with the software. For the pseudo-random points, an algorithm randomly chooses approximately 20 points in the union of the $5 \mathrm{~mm}$ border around the inhale and exhale right lung and approximately 10 points in the union of the interior of the inhale and exhale right lungs.

To register two datasets, control points in the homologous dataset are iteratively perturbed using the Nelder-Mead simplex algorithm to deform the homologous dataset to maximize the mutual information between it and the reference dataset. $^{25}$ The control points in the reference dataset remained fixed, while the homologous control points are allowed to move after each iteration. The distance the homologous points can move in any direction during the first iteration is specified by the user, while subsequent allowed perturbations are chosen by the optimization algorithm. Since, the initial control points are assumed to be an educated guess of the alignment, a length of $1 \mathrm{~cm}$ was chosen for the initial perturbation distance. The registration procedure ends when the change in MI after three consecutive 


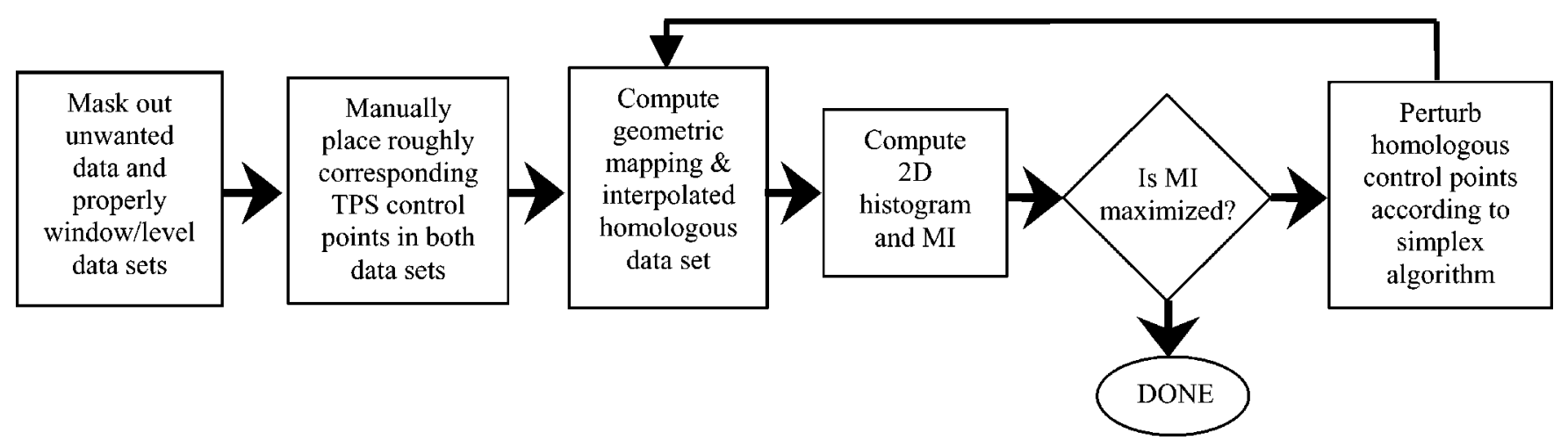

FIG. 2. Flow chart representing the MI-based image registration method using thin-plate splines.

iterations has not exceeded a specified threshold. In this study, a threshold of 0.01 was chosen for the convergence criteria. A flow chart representing the registration procedure is shown in Fig. 2.

\section{Data analysis}

To quantitatively analyze the accuracy of the image alignment, the position of known features in the inhale and exhale CT scan data was analyzed. An observer chose six landmarks within the right lung per patient. Landmarks included vascular as well as bronchial bifurcations and an effort was made to distribute the landmarks uniformly in the right lung volume for each patient. An effort was made to choose control points that did not correspond with landmarks as to not bias the assessment of the registration accuracy. The average distance to the closest reference control point from an exhale landmark was $2.8 \mathrm{~cm} \pm 1.0 \mathrm{~cm}$. An example of a vascular bifurcation landmark is shown in Fig. 3.

After choosing landmarks for a pair of CT scans, the computed TPS transform obtained from the image registration was applied to the exhale landmark coordinates to obtained predicted inhale landmark coordinates. These predicted inhale coordinates were compared to the actual inhale coordinates chosen on the inhale CT scan to quantify the accuracy of the image registration. The differences between actual and predicted inhale landmark positions are given as

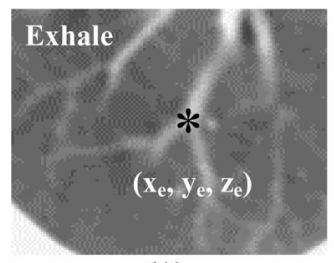

(A)

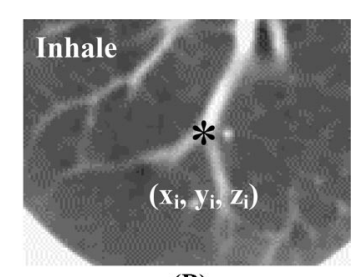

(B)
FIG. 3. An example vascular bifurcation landmark on the (a) exhale CT dataset and (b) inhale CT dataset.

$$
\begin{aligned}
D_{k}(i, j)= & \left(d_{k, \text { predicted_inhale }}(i, j)-d_{k, \text { inhale }}(i, j)\right), \\
& k=\text { RL,AP,IS, }, \quad i=1 \cdots 11, \quad j=1 \cdots 6,
\end{aligned}
$$

where $k$ is the RL, AP, or IS axis, $d_{k \text {,inhale }(i, j)}$ is the actual inhale landmark position in the $k$ direction for the $j$ th landmark of patient $I$, and $d_{k \text {,predicted_inhale }(i, j)}$ is the predicted inhale coordinate for the $k$ axis for the $j$ th landmark in patient $i$. The difference between exhale and inhale coordinates was used to obtain the extent of breathing motion in each direction at the landmark position as

$$
\begin{aligned}
B_{k}(i, j)= & \left(d_{k, \text { exhale }}(i, j)-d_{k, \text { inhale }}(i, j)\right) \\
& k=\mathrm{RL}, \mathrm{AP}, \mathrm{IS}, \quad i=1 \cdots 11, \quad j=1 \cdots 6,
\end{aligned}
$$

where $d_{k \text {,exhale }(i, j)}$ is the $k$ coordinate of the $j$ th landmark's exhale position for patient $i$, and $d_{k, \text { inhale }(i, j)}$ is again the corresponding inhale landmark position in the $k$ direction for the $j$ th landmark of patient $i$.

The reproducibility of choosing the landmarks was also measured. An observer was shown a chosen landmark on the exhale dataset and was asked to locate the corresponding landmark on the inhale dataset. Four trials, a minimum of eight hours apart, were done on 10 different landmarks. This included 2 separate bifurcations on five different patients. Reproducibility results were calculated as the standard deviation of differences between consecutively chosen landmark positions in each direction for the 10 landmarks.

To determine the significance of deformation in aligning inhale to exhale lung models, affine registrations were done using rotate-translate, rotate-translate-scale, and a full affine transformation (rotate-translate-scale-shear). These alignments used the same initial 30 control points for an input to affine registration. When the registration procedure met the convergence criteria, the same landmark position analysis was applied to estimate accuracy. 


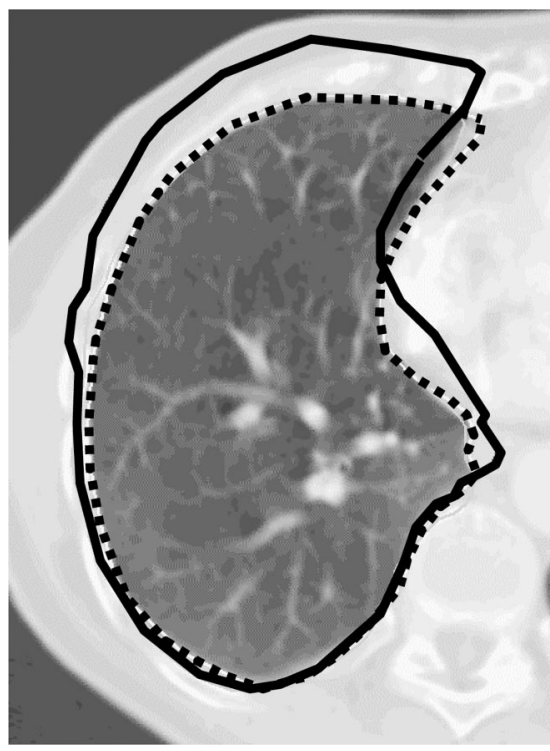

FIG. 4. A visual representation of the TPS registration accuracy. Shown is an axial exhale CT slice with the actual inhale CT right lung contour in black and the deformed inhale contour after TPS mapping to the exhale position (dashed line).

\section{RESULTS}

MI-based alignment using thin-plate splines between inhale and exhale CT models of the lung was performed on 11 pairs of CT scans using 30 control points placed as described. In addition, three affine alignments, including rotate-translate (RT), rotate-translate-scale (RTS), and rotate-translate-scale-shear (RTSS), were performed on each pair of datasets with the same 30 control points. Bronchial and vascular bifurcations were chosen for a quantitative analysis of registration accuracy. The magnitude of breathing motion was measured for each landmark to obtain significance information for the registration accuracy.

The reproducibility of choosing bronchial and vessel bifurcations was $0.46 \mathrm{~mm}$ in the RL direction and $0.48 \mathrm{~mm}$ in the AP direction. The IS direction reproducibility is on the order of the bifurcation size because bifurcations were specifically chosen to only be visible on one axial CT slice in each dataset. Therefore, if analyzed, the error in the IS direction would be $0.0 \mathrm{~mm}$ because, when repeatedly choosing the same bifurcation, the observer would always choose the same axial slice. With a slice thickness of $5 \mathrm{~mm}$, the maximum positioning error would be $5 \mathrm{~mm}$, although it would generally be much smaller than this. The magnitude of breathing motion was determined by taking the difference between landmark positions at exhale and inhale. The mean magnitude of breathing motion was $-0.4 \mathrm{~mm}$ (SD $=2.7 \mathrm{~mm})$ in the RL direction, $8.1 \mathrm{~mm}(\mathrm{SD}=6.6 \mathrm{~mm})$ in the AP direction, and $3.2 \mathrm{~mm}(\mathrm{SD}=8.6 \mathrm{~mm})$ in the IS direction.

The accuracy of alignment in each direction was calculated by taking the standard deviation of the differences between predicted and actual inhale positions for all landmarks and found to be $1.7 \mathrm{~mm}$ in the RL direction, $3.1 \mathrm{~mm}$ in the AP direction, and $3.6 \mathrm{~mm}$ in the IS direction. A paired $t$-test was performed to determine if the alignment accuracy was significant with regard to the magnitude of breathing motion. The alignment accuracy in the RL direction could not be distinguished from the magnitude of breathing movement with a $p$-value of 0.4. Alignment accuracy in the AP and IS directions was significantly discernable from the breathing motion with $p$-values of $4 \times 10^{-10}$ in the AP direction and 0.007 in the IS direction. There did not appear to be any systematic registration errors, with the average distance from predicted to actual inhale positions being $0.0 \mathrm{~mm}$ in the RL direction, $-0.5 \mathrm{~mm}$ in the AP direction, and $0.4 \mathrm{~mm}$ in the IS direction. Figure 4 shows an actual axial exhale CT image with drawings representing the original inhale CT contour (solid line) as well as the deformed inhale contour after mapping to the exhale position (dashed line).

Table I summarizes the accuracy results for the TPS alignments. Given are the mean and standard deviation of $D_{\mathrm{RL}}$, $D_{\mathrm{AP}}$, and $D_{\mathrm{IS}}$ values for each patient, as given in Eq. (1). In the table, the subscript $i$ denotes the values are for patient $i$, and the final row simply gives the mean and standard deviation for all landmarks.

TABLE I. The mean and standard deviation of the differences between the predicted and actual coordinates of landmarks at inhale based on deformable image registration for each patient $(\mathrm{cm})$. The final row is the mean and standard deviation taken over all landmarks (66).

\begin{tabular}{|c|c|c|c|c|c|c|}
\hline \multirow{2}{*}{$\begin{array}{c}\text { Patient No. } \\
1\end{array}$} & \multicolumn{2}{|c|}{$\bar{D}_{\mathrm{RL}, i}\left(\sigma_{D_{\mathrm{RL}, i}}\right)$} & \multicolumn{2}{|c|}{$\bar{D}_{\mathrm{AP}, i}\left(\sigma_{D_{\mathrm{AP}, i}}\right)$} & \multicolumn{2}{|c|}{$\bar{D}_{\mathrm{IS}, i}\left(\sigma_{D_{\mathrm{IS}, i}}\right)$} \\
\hline & 0.06 & $(0.16)$ & 0.10 & $(0.23)$ & 0.13 & $(0.12)$ \\
\hline 2 & -0.07 & $(0.09)$ & -0.22 & $(0.23)$ & -0.24 & $(0.35)$ \\
\hline 3 & -0.04 & $(0.20)$ & 0.32 & $(0.46)$ & 0.17 & $(0.49)$ \\
\hline 4 & 0.09 & $(0.10)$ & -0.03 & $(0.38)$ & 0.00 & $(0.36)$ \\
\hline 5 & 0.12 & $(0.30)$ & -0.08 & $(0.29)$ & -0.09 & $(0.36)$ \\
\hline 6 & -0.01 & $(0.16)$ & -0.09 & $(0.20)$ & 0.11 & $(0.44)$ \\
\hline 7 & -0.05 & $(0.08)$ & 0.03 & $(0.14)$ & 0.02 & $(0.19)$ \\
\hline 8 & 0.01 & $(0.21)$ & -0.28 & $(0.34)$ & 0.24 & $(0.36)$ \\
\hline 9 & 0.03 & $(0.14)$ & -0.23 & $(0.34)$ & 0.26 & $(0.33)$ \\
\hline 10 & -0.03 & $(0.07)$ & 0.01 & $(0.17)$ & -0.13 & $(0.28)$ \\
\hline 11 & -0.07 & $(0.19)$ & -0.11 & $(0.23)$ & -0.03 & $(0.44)$ \\
\hline All patients & 0.00 & $(0.17)$ & -0.05 & $(0.31)$ & 0.04 & $(0.36)$ \\
\hline
\end{tabular}




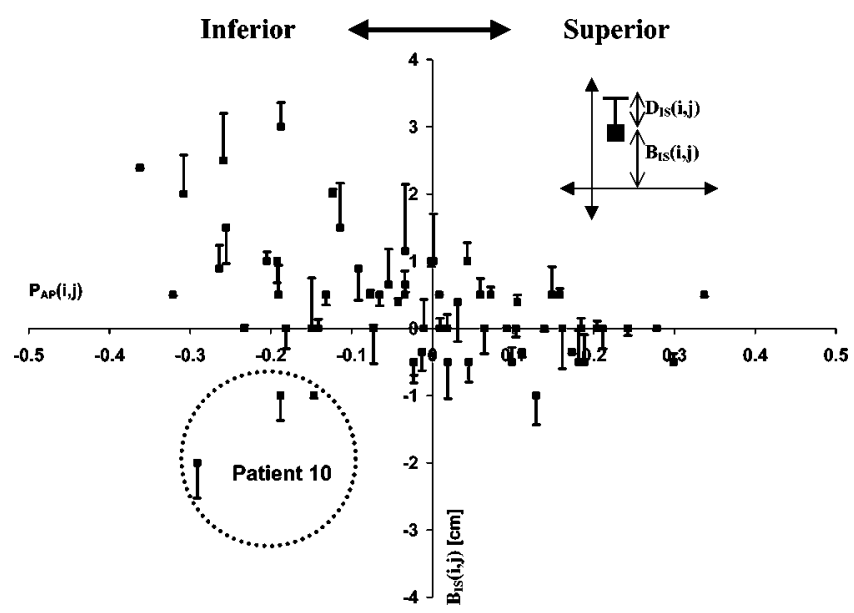

FIG. 5. The magnitude of breathing motion as compared to alignment accuracy in the IS direction for the MI-based TPS registration between exhale and inhale CT lung models. The distance to the square marker represents the difference between exhale and inhale IS coordinates of the landmark position. The length of the corresponding line represents the distance between predicted and actual inhale positions. Note that patient $10 \mathrm{did}$ not following the breathing trend of the other 10 patients.

Plots illustrating the landmark displacement from exhale to inhale versus the accuracy of alignment are shown in Figs. 5-7. Each data point represents a value of $B_{k}$, as described in Eq. (2), and the corresponding bar represents the value of $D_{k}$ for that respective landmark. The $x$-axis is the fractional position of the coordinate from the median position of the right lung in the given direction, defined as

$$
\begin{aligned}
& P_{k}(i, j)=\frac{d_{k, \text { exhale }}(i, j)-\left[\frac{\operatorname{Min} E_{k}(i)+\operatorname{Max} E_{k}(i)}{2}\right]}{\left|\operatorname{Min} E_{k}(i)-\operatorname{Max} E_{k}(i)\right|}, \\
& k=\mathrm{RL}, \mathrm{AP}, \mathrm{IS}, \quad i=1 \cdots 11, \quad j=1 \cdots 6,
\end{aligned}
$$

where $d_{k, \text { exhale }(i, j)}$ is the $k$ coordinate of the $j$ th landmark's exhale position for patient $i$, and $\operatorname{Min} E_{k}(i)$ and $\operatorname{Max} E_{k}(i)$ are the minimum and maximum extent of the right lung volume on the exhale CT scan on the $k$ axis for patient $i$. The standard deviations of the $D_{\mathrm{RL}}, D_{\mathrm{AP}}$, and $D_{\mathrm{IS}}$ values for all landmarks were $3.8 \mathrm{~mm}, 6.3 \mathrm{~mm}$, and $9.0 \mathrm{~mm}$, respectively, when allowing the inhale dataset to rotate or translate only. Adding scaling capabilities changed the accuracy to $4.0 \mathrm{~mm}$, $5.2 \mathrm{~mm}$, and $9.2 \mathrm{~mm}$ in the RL, AP, and IS directions, respectively. A full affine alignment including shearing capabilities resulted in standard deviations between differences in predicted and actual inhale coordinates of $2.6 \mathrm{~mm}, 4.6 \mathrm{~mm}$, and $5.3 \mathrm{~mm}$ in the RL, AP, and IS directions. Table II summarizes the accuracy results for the TPS versus affine alignments. Also included are the results of the TPS registrations for the corresponding inhale-exhale control point pairs, and pseudo-randomly placed control points. There does appear to be an advantage to placing corresponding control points on both datasets as compared to the current method, however, the pseudo-random method appears to create a systematic error in the alignment the AP and IS directions where the deformation is mainly in one direction.

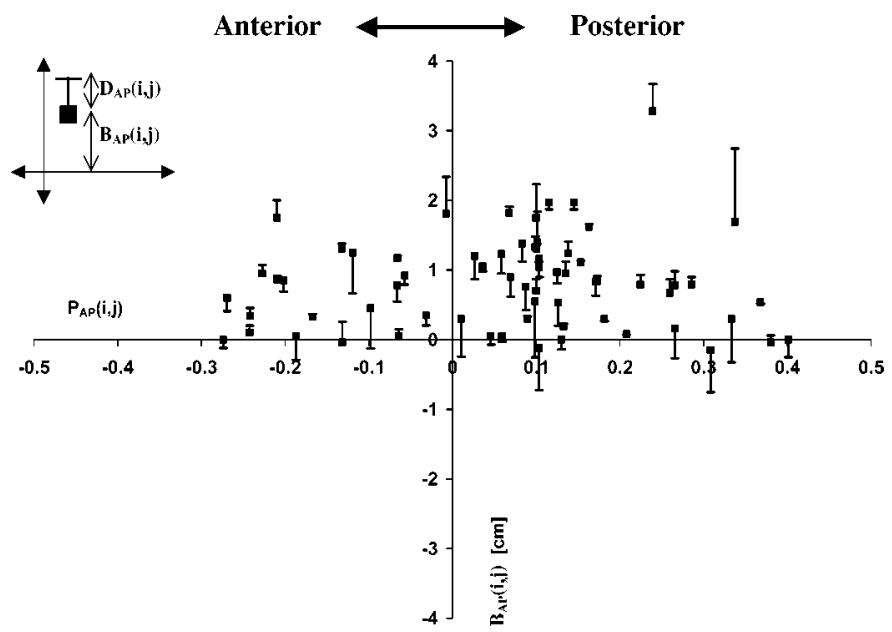

FIG. 6. The magnitude of breathing motion as compared to alignment accuracy in the AP direction for the MI-based TPS registration between exhale and inhale CT lung models. The distance to the $d x$ marker represents the difference between exhale and inhale positions of the landmark. The length of the corresponding line represents the distance between predicted and actual inhale positions.

\section{DISCUSSION}

The described method of MI-based image registration using thin-plate splines for interpolation with iterative alignment of control points based on the simplex algorithm is an accurate registration tool for use in the development of dynamic models of the lung. The use of landmark comparisons to test alignment accuracy appears to be sufficient as the reproducibility of choosing landmarks is less than $0.5 \mathrm{~mm}$ in both the RL and AP directions.

Breathing motion was estimated by comparing the positions of the landmarks at exhale and inhale and found, on average, to be $-0.4 \mathrm{~mm}(\mathrm{SD}=2.7 \mathrm{~mm})$ in the RL direction, $8.1 \mathrm{~mm}(\mathrm{SD}=6.6 \mathrm{~mm})$ in the AP direction, and $3.2 \mathrm{~mm}$ $(\mathrm{SD}=8.6 \mathrm{~mm})$ in the IS direction. One patient that exhibited

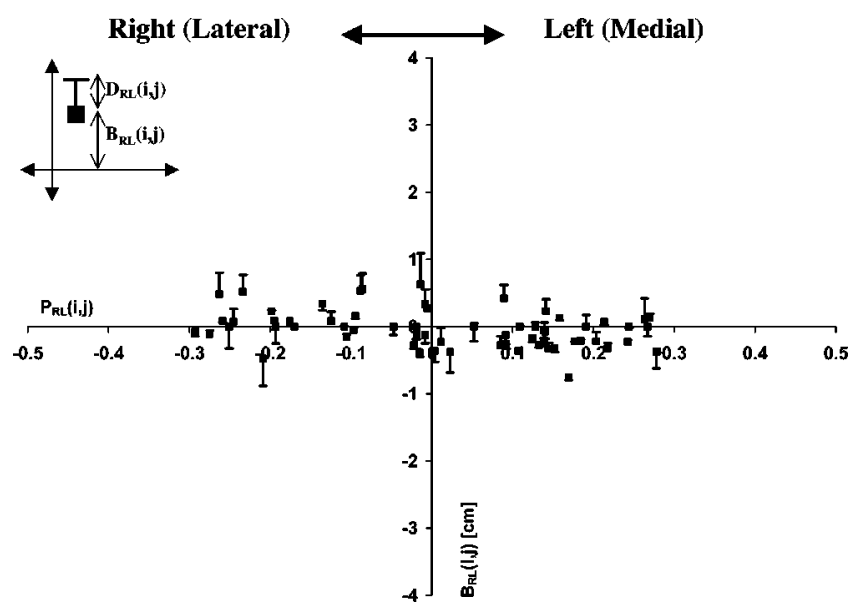

FIG. 7. The magnitude of breathing motion as compared to alignment accuracy in the RL direction for the MI-based TPS registration between exhale and inhale CT lung models. The distance to the $d x$ marker represents the difference between exhale and inhale positions of the landmark. The length of the corresponding line represents the distance between predicted and actual inhale positions. 
TABLE II. The mean and standard deviation of the differences between the predicted and actual coordinates of landmarks at inhale for thin-plate spline and affine registrations. Affine registration include rotate-translate (RT), rotate-translate-scale (RTS), and rotate-translate-scale-shear (RTSS). Also included are results from TPS registrations for the corresponding control points pairs and pseudo-randomly placed control points. Measurements are given in centimeters.

\begin{tabular}{lrrrrrr}
\hline \hline Alignment & \multicolumn{2}{c}{$\bar{D}_{\mathrm{RL}}\left(\sigma_{D_{\mathrm{RL}}}\right)$} & \multicolumn{2}{c}{$\bar{D}_{\mathrm{AP}}\left(\sigma_{D_{\mathrm{AP}}}\right)$} & \multicolumn{2}{c}{$\bar{D}_{\mathrm{IS}}\left(\sigma_{D_{\mathrm{IS}}}\right)$} \\
\hline TPS & 0.00 & $(0.17)$ & -0.05 & $(0.31)$ & 0.04 & $(0.36)$ \\
Affine-RT & -0.08 & $(0.38)$ & -0.08 & $(0.63)$ & 0.11 & $(0.90)$ \\
Affine-RTS & -0.10 & $(0.40)$ & 0.11 & $(0.52)$ & -0.11 & $(0.92)$ \\
Affine-RTSS & -0.06 & $(0.26)$ & 0.17 & $(0.46)$ & -0.07 & $(0.53)$ \\
TPS (manual) & 0.00 & $(0.19)$ & -0.01 & $(0.24)$ & -0.06 & $(0.34)$ \\
TPS (random) & -0.04 & $(0.21)$ & 0.19 & $(0.54)$ & 0.16 & $(0.68)$ \\
\hline \hline
\end{tabular}

uncharacteristic breathing motion in the IS direction is the reason for the large standard deviation in that direction. A more accurate assessment of breathing motion would be possible with the definition of more than six landmarks, but since the focus of this study was to evaluate the use of thinplate splines to model lung deformation, only a small number of landmarks were used to provide a first approximation. It is possible that a more in depth investigation of the breathing motion would allow a better characterization of the alignment tool in the RL direction, however, due to the small landmark displacement observed in this direction, even the most accurate alignment may be clinically irrelevant.

We are investigating the ability of our alignment tool to register the entire thorax and produce intermediate breathing states. For this to be clinically useful, more information on respiratory behavior similar to that acquired by Seppenwoolde et al. would be essential. ${ }^{23}$

A deformable registration tool such as the one described is necessary to model the motion of the lung between inhale and exhale. This is evident by the inability of affine registration to reach the same accuracy of alignment as deformable registration. A full affine alignment showed promise, but was still less accurate than the thin-plate spline registration. This improvement may be clinically unimportant in some situations, and the location of the tumor as well as the extra time needed for deformable alignment should be considered when choosing a registration method in the lung. Improvement on the deformable alignment model will be investigated by testing different interpolants such as B-splines as well as different evaluation metrics. Early work using B-splines indicates that they may offer more control in the registration and mean squared error may be superior to mutual information at a metric to evaluate CT to CT image registration. Different combinations of interpolants and metrics may be appropriate for specific anatomic sites and organs.

\section{CONCLUSIONS}

The technique shown for mutual information-based image alignment using thin-plate splines shows promise for the alignment of inhale to exhale CT models of the lung using 30 control points initially placed manually and then iteratively adjusted by the simplex algorithm. The sufficient accuracy of alignment was shown in the AP and IS direction of $3.1 \mathrm{~mm}$ and $3.6 \mathrm{~mm}$, respectively. Alignment accuracy in the RL direction was similar $(1.7 \mathrm{~mm})$, but was unable to be distinguished from the small magnitude of breathing motion in that direction. Improved accuracy of this method over affine registrations was also observed although the clinical significance of the improvement should be investigated, taking into account the location of the tumor as well as the increased time needed for deformable registration. Work is continuing in deformable registration interpolants and different evaluation metrics.

\section{ACKNOWLEDGMENTS}

The authors would like to thank Dr. Charles Meyer from the department of Radiology for collaboration on implementation of the deformable alignment code as well as Kristy Brock and Rojano Kashani for their efforts relating to this work. This work was supported in part by NIH P01CA59827.

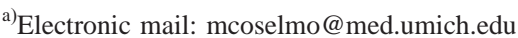

${ }^{1}$ ICRU Report 62, "Prescribing, recording, and reporting photon beam therapy" (supplement to ICRU Report 50), ICRU, Bethesda, MD, 1998.

${ }^{2}$ A. Lujan et al., "A method for incorporating organ motion due to breathing into 3D dose calculations," Med. Phys. 26, 715-720 (1999).

${ }^{3}$ T. Craig et al., "Limitations of a convolution method for modeling geometric uncertainties in radiation therapy. I. The effect of shift invariance," Med. Phys. 30, 2001-2011 (2003).

${ }^{4}$ T. Craig et al., "Limitations of a convolution method for modeling geometric uncertainties in radiation therapy. II. The effect of a finite number of fractions," Med. Phys. 30, 2012-2020 (2003).

${ }^{5}$ I. Chetty et al., "A fluence convolution method to account for respiratory motion in three-dimensional dose calculations of the liver: A Monte Carlo study," Med. Phys. 30, 1776-1780 (2003).

${ }^{6} \mathrm{~W}$. Beckham et al., "A fluence convolution method to calculate radiation therapy dose distributions that incorporate random set-up error," Phys. Med. Biol. 47, 3464-3473 (2002).

${ }^{7} \mathrm{G}$. Hugo et al., "The effects of tumor motion on planning and delivery of respiratory-gated IMRT," Med. Phys. 30, 1052-1066 (2003).

${ }^{8}$ R. Wagman et al., "Respiratory gating for liver tumors: Use in dose escalation,” Int. J. Radiat. Oncol., Biol., Phys. 55, 659-668 (2003).

${ }^{9}$ T. Neicu et al., "Synchronized moving aperture radiation therapy (SMART): average tumour trajectory for lung patients," Phys. Med. Biol. 48, 587-598 (2003)

${ }^{10} \mathrm{~F}$. Maes et al., "Multimodality image registration by maximization of mutual information," IEEE Trans. Med. Imaging 16, 187-198 (1997).

${ }^{11} \mathrm{C}$. Meyer et al., "Demonstration of accuracy and clinical versatility of mutual information for automatic multimodality image fusion using affine and thin-plate spline warped geometric deformations," Med. Image Anal
} 
1, 196-206 (1997).

${ }^{12} \mathrm{~K}$. Rohr et al., "Landmark-based elastic registration using approximating thin-plate splines," IEEE Trans. Med. Imaging 20, 526-534 (2001).

${ }^{13} \mathrm{H}$. Johnson et al., "Consistent landmark and intensity-based image registration," IEEE Trans. Med. Imaging 21, 450-461 (2002).

${ }^{14} \mathrm{G}$. Christensen et al., "Image-based dose planning of intracavitary brachytherapy: Registration of serial-imaging studies using deformable anatomic templates," Int. J. Radiat. Oncol., Biol., Phys. 51, 227-243 (2001).

${ }^{15} \mathrm{M}$. Jacobs et al., "Registration and warping of magnetic resonance images to histological sections," Med. Phys. 26, 1568-1578 (1999).

${ }^{16} \mathrm{D}$. Lockman et al., "Estimating the dose variation in a volume of interest with explicit consideration of patient geometric variation," Med. Phys 27, 2100-2108 (2000).

${ }^{17} \mathrm{D}$. Yan et al., "Organ/patient geometric variation in external beam radiotherapy and its effects," Med. Phys. 28, 593-602 (2003).

${ }^{18} \mathrm{C}$. Meyer et al., "Method for quantifying volumetric lesion change in internal liver CT examinations," IEEE Trans. Med. Imaging 22, 776-781 (2003).

${ }^{19} \mathrm{~K}$. Brock et al., "Automated generation of a four-dimensional model of the liver using warping and mutual information," Med. Phys. 30, 11281133 (2003).

${ }^{20} \mathrm{~K}$. McLeish et al., "A study of the motion and deformation of the heart due to respiration," IEEE Trans. Med. Imaging 21, 1142-1150 (2002).

${ }^{21} \mathrm{~K}$. Brock et al., "Inclusion of organ deformation in dose calculations," Med. Phys. 30, 290-295 (2003).

${ }^{22} \mathrm{Q}$. Chen et al., "Fluoroscopic study of tumor motion due to breathing: Facilitating precise radiation therapy for lung cancer patients," Med. Phys. 28, 1850-1856 (2001).

${ }^{23} \mathrm{Y}$. Seppenwoolde et al., "Precise and real-time measurement of 3D tumor motion in lung due to breathing and heartbeat, measured during radiotherapy," Int. J. Radiat. Oncol., Biol., Phys. 53, 822-834 (2002).

${ }^{24}$ J. Balter et al., "Uncertainties in CT-based radiation therapy treatment planning associated with patient breathing," Int. J. Radiat. Oncol., Biol., Phys. 36, 167-174 (1996).

${ }^{25}$ W. Press et al., Numerical Recipes in C: The Art of Scientific Computing (Cambridge University Press, Cambridge, 1988).

${ }^{26}$ J. Pluim et al., "Mutual-information-based registration of medical images: a survey," IEEE Trans. Med. Imaging 22, 986-1004 (2003). 